\title{
Multi-instrument observations of the electric and magnetic field structure of omega bands
}

\author{
J. A. Wild ${ }^{1}$, T. K. Yeoman ${ }^{1}$, P. Eglitis ${ }^{2,3}$, H. J. Opgenoorth ${ }^{3,4}$ \\ ${ }^{1}$ Department of Physics and Astronomy, University of Leicester, University Road, Leicester, LE1 7RH, UK \\ 2 Department of Astronomy, Uppsala University, St. Olofsgatan 10, Box 256, S-751 05 Uppsala, Sweden \\ ${ }^{3}$ Finnish Meteorological Institute, Box 503, SF-00101, Helsinki, Finland \\ ${ }^{4}$ Swedish Institute of Space Physics, Uppsala Division, S-755 91 Uppsala, Sweden
}

Received: 16 June 1999 / Revised: 25 August 1999 / Accepted: 27 August 1999

\begin{abstract}
High time resolution data from the CUTLASS Finland radar during the interval 01:30-03:30 UT on 11 May, 1998, are employed to characterise the ionospheric electric field due to a series of omega bands extending $\sim 5^{\circ}$ in latitude at a resolution of $45 \mathrm{~km}$ in the meridional direction and $50 \mathrm{~km}$ in the azimuthal direction. E-region observations from the STARE Norway VHF radar operating at a resolution of $15 \mathrm{~km}$ over a comparable region are also incorporated. These data are combined with ground magnetometer observations from several stations. This allows the study of the ionospheric equivalent current signatures and height integrated ionospheric conductances associated with omega bands as they propagate through the field-of-view of the CUTLASS and STARE radars. The high-time resolution and multi-point nature of the observations leads to a refinement of the previous models of omega band structure. The omega bands observed during this interval have scale sizes $\sim 500 \mathrm{~km}$ and an eastward propagation velocity $\sim 0.75 \mathrm{~km} \mathrm{~s}^{-1}$. They occur in the morning sector ( 05 MLT), simultaneously with the onset/intensification of a substorm to the west during the recovery phase of a previous substorm in the Scandinavian sector. A possible mechanism for omega band formation and their relationship to the substorm phase is discussed.
\end{abstract}

Key words. Ionosphere (auroral ionosphere; electric fields and currents) $\cdot$ Magnetospheric physics (magnetosphere-ionosphere interactions)

\section{Introduction}

Omega bands are a distinct class of auroral form that generally occur in the morning sector during the

Correspondence to: J. A. Wild

E-mail: jaw11@ion.le.ac.uk recovery phase of magnetospheric substorm (Akasofu, 1974; Saito, 1978; Rostoker and Barichello, 1980). They are characterised by bright regions extending from the poleward boundary of the aurora separated by a dark region that loosely resembles an inverted Greek letter $\Omega$. In many cases the bright protruding regions, originally described as "torches" by Akasofu and Kimball (1963), also resemble the letter $\Omega$ and the term "omega band" is used to describe auroral features with either characteristic. Omega bands have been observed to propagate in the eastward direction at velocities $\sim 1 \mathrm{~km} \mathrm{~s}^{-1}$ (Lühr and Schlegel, 1994) and have also been observed in both hemispheres simultaneously (Mravlag et al., 1991). As they travel eastward the spatial structure of omega bands remains relatively unchanged and observations of omega bands are usually accompanied by azimuthal component magnetic disturbances. Studies by various authors (Saito, 1978; Kawasaki and Rostoker, 1979; Opgenoorth et al., 1983; Buchert et al., 1988) have concluded that the observation of a Ps 6 pulsation (period 4-40 min) implies the existence of an auroral omega band. In addition, previous authors have linked Ps 6 pulsations to the propagation of vortical Hall currents associated with field-aligned currents (FACs) (e.g. Lühr and Schlegel, 1994) although the mechanism by which these FACs arise is still uncertain.

The stable nature of the omega band structure combined with rapid propagation in the azimuthal direction implies that extended networks of magnetometer stations are particularly suitable for the study of this phenomena. Equivalent currents calculated from ground magnetometer stations reveal much information regarding the spatial extent of the auroral features and the current systems contained within them.

High frequency (HF) and very high frequency (VHF) coherent scatter radars measure signals backscattered off magnetic field-aligned plasma irregularities. These provide measurements of line-of-sight (l-o-s) ionospheric convection velocities from which estimates of ionospheric electric fields can be derived over a very large field-ofview at relatively high temporal resolution (see Sect. 2). 
Furthermore, by combining the ionospheric electric field strength inferred from radar observations and magnetic field measurements from ground magnetometers within the radar field-of-view it is possible to make estimates of the ionospheric Hall conductance at several points inside the region of interest. Whilst this technique does not readily yield absolute measurements of conductance such as those obtained using incoherent scatter radars (e.g. Buchert et al., 1988), the estimates can be made over a greatly extended area when compared with the incoherent scatter approach. The resulting distribution of high and low conductance contributes further to the understanding of omega band structure and electrodynamics.

In this work, we present observations of an interval of intense Ps 6 pulsations with the Co-operative UK Twin Located Auroral Sounding System (CUTLASS) Finland HF radar, the Scandinavian Twin Auroral Radar Experiment (STARE) Norway VHF radar and elements of the Greenland coastal and International Monitor for Auroral Geomagnetic Effects (IMAGE) magnetometer networks. The simultaneous multi-instrument observations allow us to deduce a two-dimensional description of the omega bands in terms of magnetic perturbations, FACs, ionospheric electric fields, Hall conductances and equivalent currents. Finally, various proposed formation mechanisms are considered and the relationship between omega bands and the substorm phases are discussed.

\section{Instrumentation}

CUTLASS (Fig. 1) is a bi-static HF coherent radar, with stations in Finland and Iceland and forms part of the international SuperDARN (Super Dual Auroral Radar Network) chain of HF radars (Greenwald et al., 1995). Each radar of the system is frequency agile $(8-20 \mathrm{MHz})$, routinely measuring the line-of-sight (1-o-s) Doppler velocity and spectral width of, and the backscattered power from, ionospheric plasma irregularities. The radars form 16 beams of separation $3.24^{\circ}$. Each beam is gated into 75 range bins, each of length $45 \mathrm{~km}$ in standard operations. During standard operations the dwell time for each beam is either 7 or $3 \mathrm{~s}$, giving a full 16 -beam scan, covering $52^{\circ}$ in azimuth and over $3000 \mathrm{~km}$ in range (an area of over $3 \times 10^{6} \mathrm{~km}^{2}$ ), every 120 or $60 \mathrm{~s}$. Common volume data from the two stations can be combined to provide $\mathrm{E}$ and F-region ionospheric convection velocities perpendicular to the magnetic field. The 1-o-s ionospheric convection velocities used in this study were measured by the CUTLASS Finland radar located at Hankasalmi $\left(62.3^{\circ} \mathrm{N}, 26.6^{\circ} \mathrm{E}\right)$, Finland between 01:30-03:30 UT on 11 May 1998. During this interval the radar was operating in a non-standard scan mode. In this mode, rather than the usual anticlockwise sweep through the beams $15,14,13, \ldots, 0$ the sequence $15,9,14,9,13,9, \ldots, 1,9,0,9$ was employed. This allows the construction of full 16-beam scans at a reduced temporal resolution of $4 \mathrm{~min}$, in addition to the provision of high time resolution data $(\sim 14 \mathrm{~s})$ along a

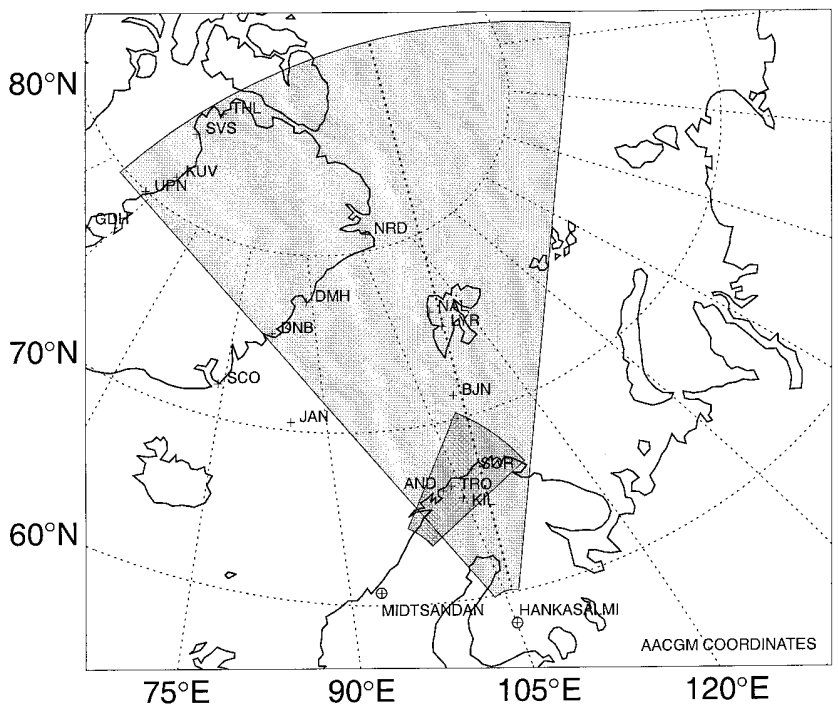

Fig. 1. Locations of the field-of-view of the CUTLASS Finland radar (pale shading), the STARE Norway radar field-of-view (dark shading) and elements of the IMAGE and Greenland magnetometer networks. Beam 9 of CUTLASS Finland which is sampled at a high rate is indicated by a dotted line

single look direction (in this case beam 9, a beam which approximately overlies the main meridional chain of the IMAGE array). The second radar of the CUTLASS pair, located at Pykkvibær $\left(63.8^{\circ} \mathrm{N}, 20.3^{\circ} \mathrm{W}\right)$, Iceland was operating in a similar mode during this interval providing one high time resolution beam in addition to reduced temporal resolution scans of the entire field-of-view. Although backscattered power from approximately $8^{\circ}$ of magnetic latitude over a period of a least 5 hours was observed by the Finland radar, no significant backscatter was observed in the same region by the Iceland radar during this interval.

The STARE system (Greenwald et al., 1978) comprises two coherent VHF radars located at Midtsandan $\left(63.4^{\circ} \mathrm{N}, 10.7^{\circ} \mathrm{E}\right)$, Norway and Hankasalmi $\left(62.3^{\circ} \mathrm{N}\right.$, $\left.26.6^{\circ} \mathrm{E}\right)$, Finland operating at $140.0 \mathrm{MHz}$ and $143.8 \mathrm{MHz}$ respectively. Each radar forms 8 beams of separation $3.6^{\circ}$ and each beam is gated into 50 range gates, each of length $15 \mathrm{~km}$ with a range to first gate of $495 \mathrm{~km}$. The beams of all CUTLASS and STARE radars are numbered in clockwise direction (0-7 in the case of STARE, $0-15$ for CUTLASS), however in contrast to the CUTLASS radars which cycles through beams sequentially to construct a scan of the field-of-view, the STARE radars scan all 8 beams simultaneously, "floodlighting" the entire field-of-view over a $20 \mathrm{~s}$ integration time. Each radar routinely measures E-region backscattered power and irregularity 1-o-s phase velocity and by combining velocity measurements from the overlapping region of the two fields of view $\left(\sim 2.3 \times 10^{5} \mathrm{~km}^{2}\right)$ it is possible to derive E-region ionospheric flow velocities perpendicular to the magnetic field. Once again, during this interval significant backscattered power was measured at one radar only (Norway). 
The majority of magnetic field observations are obtained from stations belonging to two arrays, the Greenland west coast magnetometer chain (details of the instrumentation and a description of the observatories have been given by Wilhjelm and Friis-Christensen, 1976) and the IMAGE magnetometer network (Lühr, 1994). Data from the Jan Mayen magnetometer deployed by the Tromsø Auroral Observatory have also been employed. All magnetic observations have been made by fluxgate magnetometers and data have been presented in a common co-ordinate system where $X, Y$ and $Z$ components have directions geographic north, geographic east and vertically downwards respectively.

\section{Observations}

\subsection{Ground magnetometer response}

Figure 2 presents the $X$ and $Y$ components of the local magnetic field measured at several stations of the IMAGE magnetometer array and the Jan Mayen (JAN) magnetometer of the Tromsø Auroral Observatory. These elements of the IMAGE array are located at magnetic local times approximated by $\mathrm{MLT} \approx \mathrm{UT}+2$ and Jan Mayen is located at MLT $\approx \mathrm{UT}+1$. Beginning at approximately 01:50 UT, a series of at least five Ps 6 wave-cycles with periods of approximately $18 \mathrm{~min}$ are clear in the $Y$ component magnetograms from the SOR, TRO, AND and KIL stations. Less intense pulsations are also observed at BJN. Oscillations of comparable magnitudes and periods are also apparent in the $X$ component measured at all of the IMAGE stations shown. These pulsations are not observed at JAN although both $X$ and $Y$ components are disturbed throughout the interval. At stations where the pulsations are most pronounced (e.g. TRO) the first positive deviation in the $Y$ component is significantly smaller than during later pulsations. Subsequently, the $Y$ component oscillations lead the $X$ component oscillations in phase ( $Y$ component becomes more positive as the $X$ component becomes more negative and viceversa). The maximum amplitude of the $Y$ component oscillations at TRO occurs at around 02:20 UT with a peak to peak value $\sim 150 \mathrm{nT}$. The delay between each pulsation being observed at SOR after being observed at AND suggest that the structures are propagating in an eastward direction over the Scandinavian sector with a mean velocity $\sim 0.75 \mathrm{~km} \mathrm{~s}^{-1}$. Furthermore, comparing the Ps 6 pulsation train at SOR and AND at geographic longitudes of $22.22^{\circ}$ and $16.03^{\circ}$ respectively, it is clear that ionospheric structures that gives rise to the $\mathbf{a}$

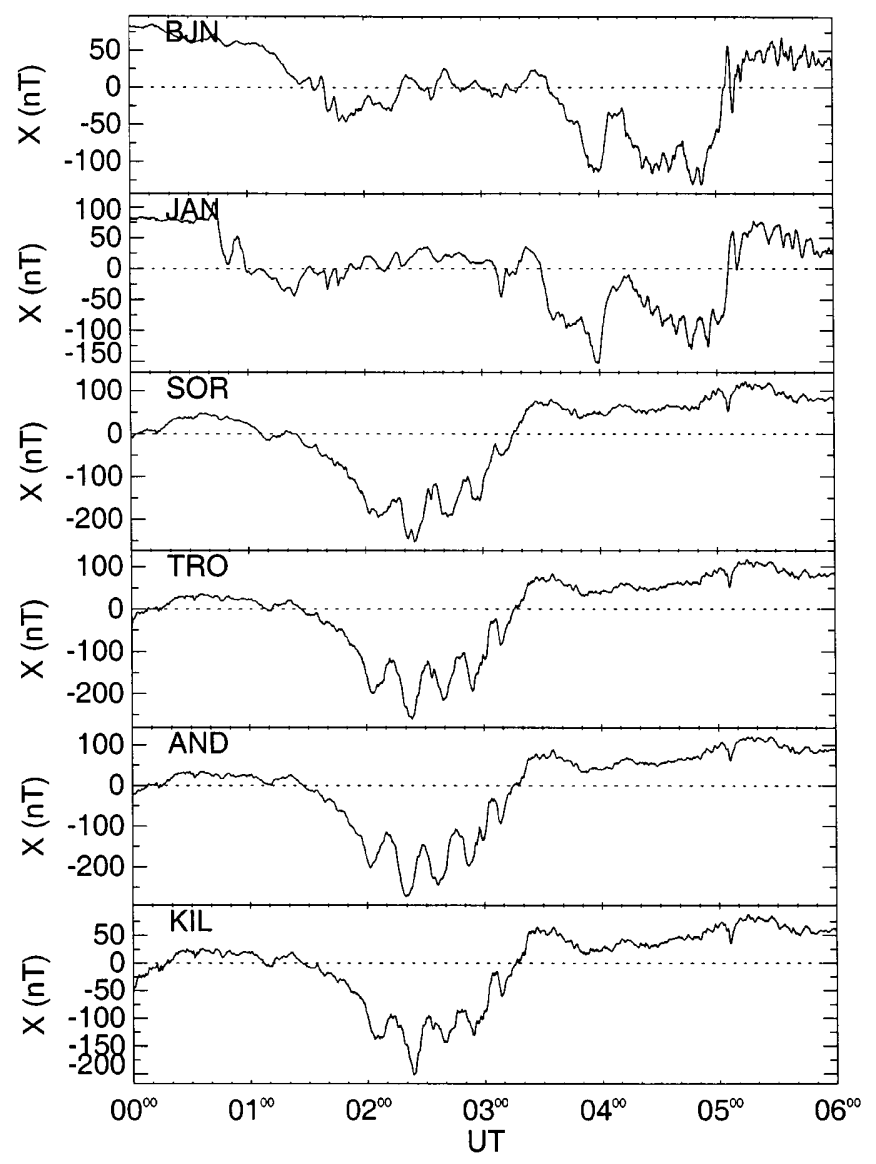

b

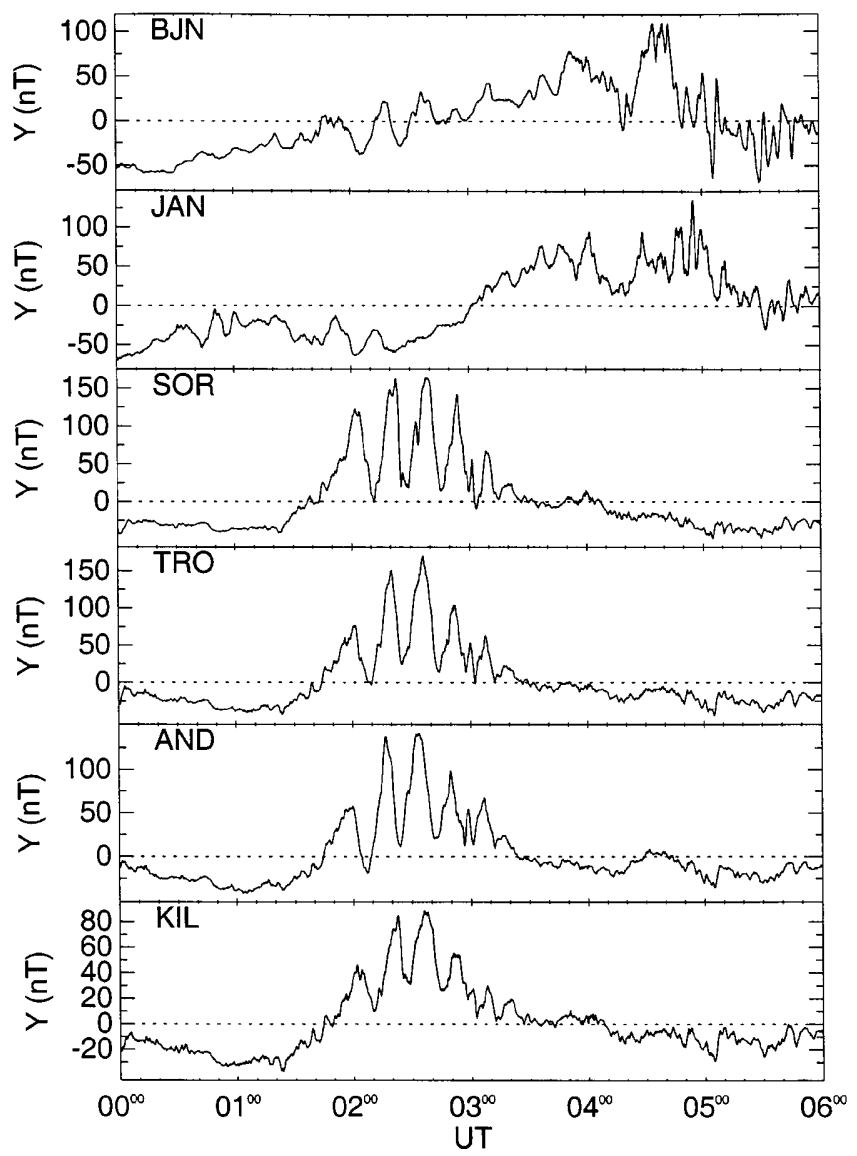

Fig. 2a, b. Ground magnetograms from the IMAGE array and Tromsø Auroral Observatory magnetometer located at Jan Mayen for the period 00:00-06:00 UT on 11 May, 1998. a $X$ components, b $Y$ components 
pulsations are coherent over the time scale of at least tens of minutes.

Observations from a selection of high-latitude magnetometers of the Greenland east-coast chain are shown in Fig. 3. At 23:08 UT ( 20:50 MLT) on 10 May, 1998, a pseudobreakup is observed at several stations of the Greenland array. At 00:12 UT on 11 May ( 22:00 MLT) a negative bay is observed in the $X$ component at NAQ and FHB. a feature indicative of intensification of a westward current and the onset of the expansion phase of a magnetospheric substorm. By 00:43 UT ( 22:30 MLT) this onset has expanded poleward to SKT. further intensification of negative bays observed at SKT at 01:35, 0150, and 03:05 UT (23:20, 23:35, and 00:50 MLT respectively) suggesting subsequent highlatitude substorm onset/intensifications. $X$ component data have been filtered between 200 and $20 \mathrm{~s}$ in order to study Pi2 pulsations (SKT only shown) and provide a reference time for the substorm onsets (Rostoker et al., 1980) and these are indicated in the figure by vertical lines. In addition, the majority of IMAGE magnetome-

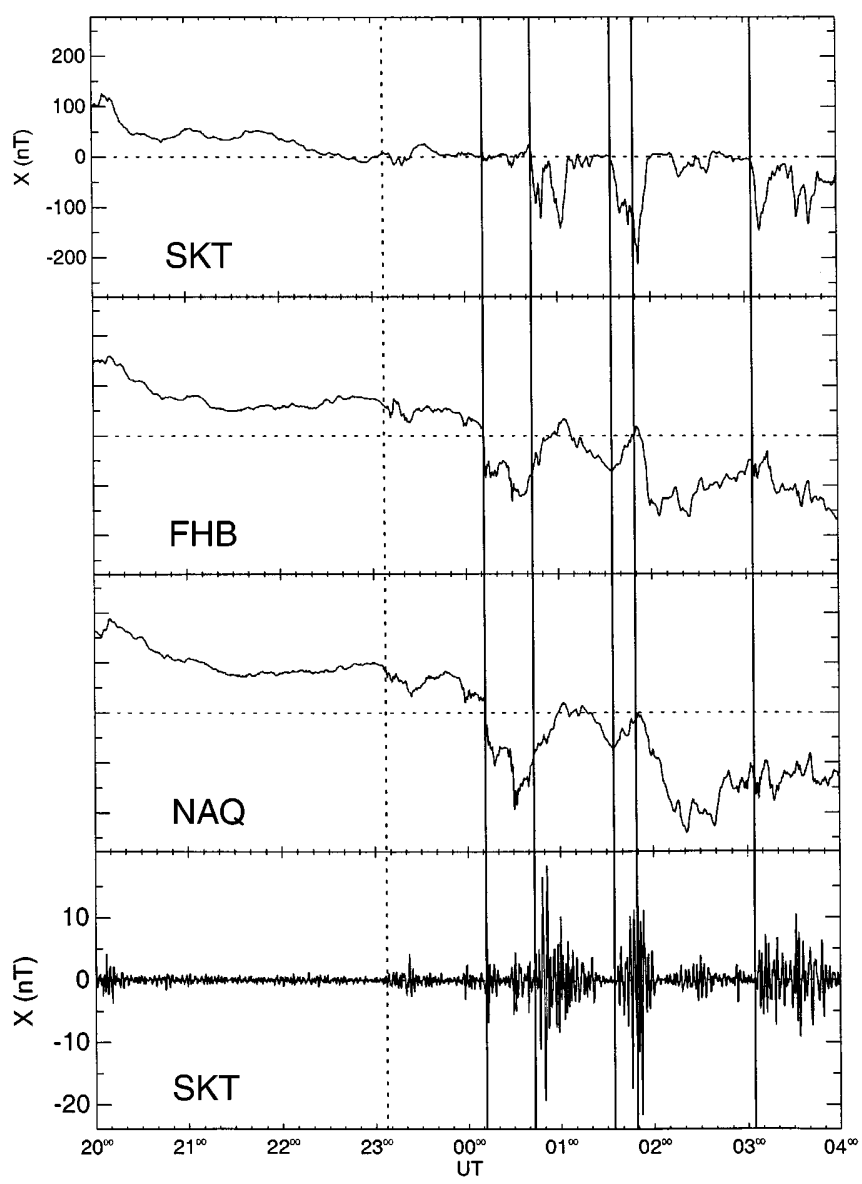

Fig. 3. Ground magnetograms from the Greenland array. $X$ component magnetograms from a subset of west coast stations for the interval 20:00 UT 10 May - 04:00 UT 11 May, 1998, are displayed. A bandpass filtered (200-20 s) trace from SKT is included in the bottom panel. Substorm expansion phase onset/intensifications are marked with solid vertical lines. The dashed vertical line at 23:08 UT indicates a pseudobreakup ters observed a significant substorm onset/intensification at 23:30 UT on the previous day (10 May, 1998 not shown).

\subsection{Radar measurements of ionospheric convection}

The line-of-sight (1-o-s) ionospheric convection velocities observed by the STARE Norway and CUTLASS Finland radars between 01:30-03:30 UT on 11 May, 1998, are presented in Fig. 4. Prior to 01:50 UT a background flow with poleward component $\leq 120 \mathrm{~m} \mathrm{~s}^{-1}$ was observed between magnetic latitudes $65^{\circ}-75^{\circ}$ by the CUTLASS radar (Fig. 4a). AT $\sim 01: 53$ UT a region of intense poleward flow $\sim 600 \mathrm{~m} \mathrm{~s}^{-1}$ developed at $\sim 71^{\circ}$ magnetic latitude and drifted $\sim 2^{\circ}$ poleward over the following $5 \mathrm{~min}$. This is immediately followed by a region of equatorward flow of velocity $\sim 240 \mathrm{~m} \mathrm{~s}^{-1}$ propagating polewards over a similar time scale and latitudinal extent. Beginning at 02:00 UT, a region of poleward flow $\sim 600 \mathrm{~m} \mathrm{~s}^{-1}$ is observed over approximately $1.5^{\circ}$ of magnetic latitude with the equatorward boundary located at $\sim 68^{\circ}$. This region was observed to move in a poleward direction at a rate of approximately $3^{\circ}$ in $10 \mathrm{~min}$ and was again followed by a region of weaker $\left(\sim 240 \mathrm{~m} \mathrm{~s}^{-1}\right)$ equatorward flow. Regions of comparable strong poleward then weak equatorward flow, always propagating away from the radar are then observed with a repetition period $\sim 18 \mathrm{~min}$. At least five such structures are clear during the interval and are marked by vertical lines at $( \pm 1 \mathrm{~min})$ : 02:00, $02: 18,02: 36,02: 53$, and 03:10 UT. In many cases a period during which no backscattered signal is observed occurs immediately after the region of enhanced flow has reached its most poleward location (e.g. 02:2502:40, magnetic latitudes $70^{\circ}-74^{\circ}$ ). By studying the elevation angle of received backscattered signal surrounding these regions, they have been determined to correspond the ionospheric F-region. It is likely that the regions of reduced backscatter correspond to regions of very low electric field and high conductance within the luminous tongue of the omega band. This would lead to the quenching of any electron density irregularities in these regions and thus the removal of the targets necessary for radar backscatter. The conductances in these regions are examined further in later sections.

E-region 1-o-s velocities measured by the STARE Norway radar (Fig. 4b) indicate a generally elevated poleward component of the background flow $\left(\sim 600 \mathrm{~m} \mathrm{~s}^{-1}\right)$ in the highest latitude region of backscatter observed by this radar $\left(\sim 69^{\circ}\right)$ when compared to F-region 1-o-s velocities measured by CUTLASS at the same latitudes. Superimposed upon this background are regions of enhanced poleward flow extending equatorward as far as $\sim 66^{\circ}$, each interval lasting $\sim 10 \mathrm{~min}$ and with a repetition period $\sim 18 \mathrm{~min}$. These occur at ( $\pm 1 \mathrm{~min}): 01: 54,02: 13,02: 32,02: 48$, and 03:04 UT and are again indicated by vertical lines. The regions of reduced backscatter observed by CUTLASS at magnetic latitudes $\geq 70^{\circ}$ are not apparent in the STARE radar data as the field-of-view does not extend to such high 


\section{a CUTLASS Finland Beam 9: vel}
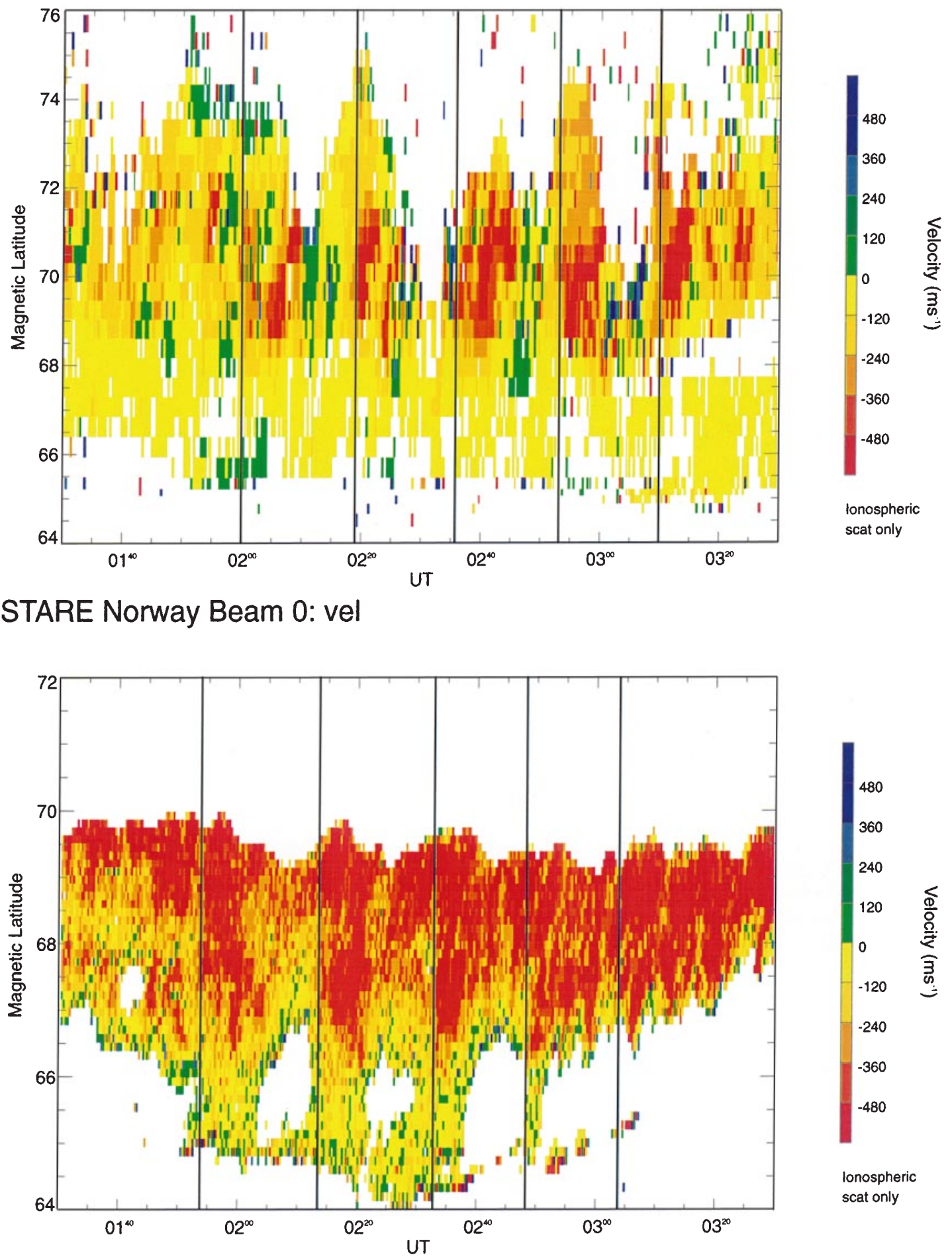

Fig. 4. a Line-of-sight velocities from beam 9 of the CUTLASS Finland radar as a function of magnetic latitude and time, showing dynamic ionospheric features associated with the auroral omega bands. Positive Doppler velocities are towards the radar (equator-

latitudes. The $\sim 5 \mathrm{~min}$ lead by which observations of enhanced poleward flow made at STARE precede those at CUTLASS is consistent with the azimuthal propaga- ward) and negative Doppler velocities are away from the radar (poleward). The timing of omega band observations are highlighted with vertical lines; b STARE Norway line-of-sight velocities measured by beam 0 as a function of magnetic latitude and time

tion time of the Ps 6 pulsations discussed above between ground magnetometers that located beneath the presented beams of the two radars. 


\subsection{Derived equivalent current features}

For some time omega bands have been associated with the ionospheric Hall current vortices that arise as a result of FACs (Opgenoorth et al., 1983; Buchert et al., 1988; Lühr and Schlegel, 1994). In order to visualise the current structure associated with the Ps 6 pulsations (and hence the omega bands) presented here, a sequence of equivalent current vectors are derived from 3 magnetometers of the IMAGE chain (Fig. 5). For an E-region current system with a spatial extent of greater than the E-region height, and assuming a horizontally uniform ionospheric conductivity, then the ground magnetic field deflections, $b$, can be related to an ionospheric equivalent current density, $J$, by

$J_{x}=-\frac{2}{\mu_{0}} b_{y} \quad J_{y}=\frac{2}{\mu_{0}} b_{x}$,

where $x$ and $y$ indicate the northward and eastward components respectively (Lühr and Schlegel, 1994). Since the omega bands appear to move eastward, according to the observed propagation delay in the magnetometer data, the current vectors have to be

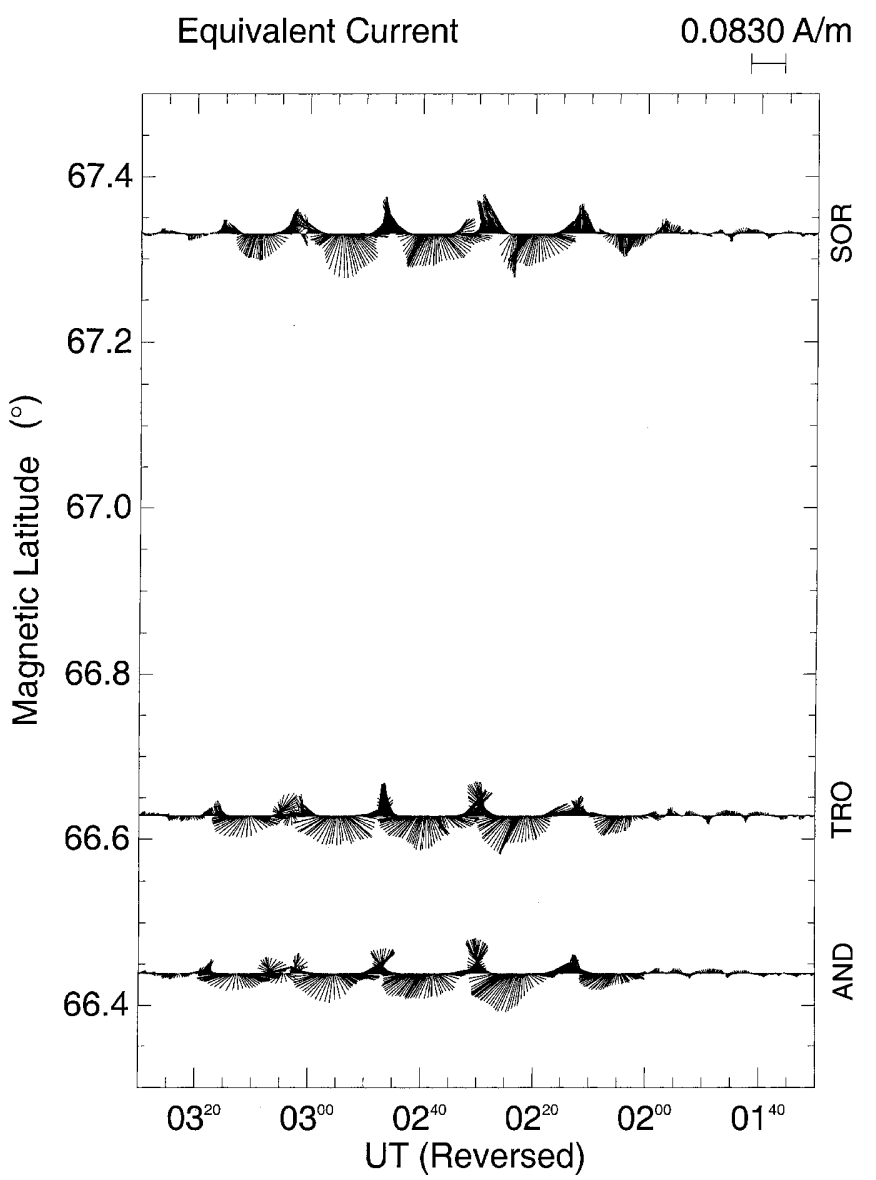

Fig. 5. Equivalent current patterns deduced from IMAGE magnetometer data. The current vectors have been plotted against a reversed time axis. This is appropriate for visualising eastward moving features. The TRO and AND time series have been shifted by 180 and 350 s respectively to account for the propagation delay between those magnetometers and SOR plotted against an inverted time scale to give a correct image of the spatial current distribution (see e.g. Lühr and Schlegel, 1994). So that direct comparisons can be made between the current distributions at various latitudes, time offsets appropriate for the propagation delay between each longitudinally separated station have been incorporated (180 and $350 \mathrm{~s}$ for TRO and AND respectively). These offsets are consistent with the time taken for each omega band to travel longitudinally between the observing magnetometer and beam 9 of the CUTCLASS radar at the speed calculated in Sect. 3.1. During the interval presented at least five pairs of vortices are observed. Each pair of vortices is associated with a region of upward and a region of downward FAC where each FAC generates a Hall current of anticlockwise and clockwise sense respectively, characteristic features of auroral omega bands. As the series of current pairs propagate eastward over the magnetometers, the measured equivalent current vectors rotate in a clockwise direction indicating that the current system is located poleward of SOR, TRO and AND. In the region between each upward/downward FAC pair, the equivalent current will be directed in an approximately equatorward direction. From Fig. 5 the equivalent current direction can be seen to achieve an equatorward pointing direction (vertically downward in figure) at the following time ( $\pm 1 \mathrm{~min}$ ): 02:04, 02:20, 02:38, 02:55, and 03:08 UT. Equivalent currents derived at BJN (not shown) rotate in an anticlockwise direction over a nearidentical timeframe implying that the observed current system is located equatorward of that station, i.e. in the region between SOR and BJN. It is just this region that is contained within the fields of view of the CUTLASS and STARE radars.

\subsection{Derived ionospheric Hall conductances}

It is possible to more closely examine the relationship between the magnetic field observed by the ground magnetometers and ionospheric speed (which is related to one component of the horizontal electric field) by combining the measured ionospheric velocities and the derived equivalent current intensity. As the equivalent Hall currents $J_{H}$ are related to the ionospheric electric field, $E$, and hence the plasma convection velocity, $v$, via the height integrated Hall conductance, $\sum_{H}$, by

$J_{H}=\sum_{H} E=\sum_{H} v B$

where $B$ is the magnetic field strength, in the present experimental arrangement an estimate of the Hall conductance may be estimated from (Lühr et al., 1993, 1996).

$\sum_{H} \approx-\frac{2}{\mu_{0}} \frac{b_{y}}{v_{x} B}$.

The 1-o-s velocities measured by the radar are assumed to have been made along a near-meridional beam direction implying 
$v_{l-o-s} \approx v_{x}$

and $b_{y}$ is simply the component of the observed magnetic field in the $Y$ direction. In order to emphasise the fluctuations in the ionospheric Hall conductances that result from omega band passage over a given location, magnetic field data have been filtered between 1200 and 300 seconds. The background magnetic field is determined by applying low pass filter (1200 s) to the original magnetic field data. This background field is then added to the filtered data to provide a time-series of magnetic field observations which although filtered, still contains information regarding the background level of the magnetic field. Finally, the absolute value of magnetic field from the quiet period prior to the Ps 6 pulsations is subtracted from the resulting time series effectively removing the quiet time and internal magnetic field variations. What remains is the magnetic field due to the enhanced electrojet and omega band structures. In the interests of consistency 1-o-s velocity data have been filtered similarly although there is no requirement to subtract the background from these absolute measurements. Figure 6 presents the parameters used to calculate $\sum_{H}$ employing data from the SOR magnetometer and CUTLASS Finland (beam 9, range gate 16). Magnetic field and 1-o-s velocities are processed in the manner described (centre 2 panels) in Fig. 6. A suitable offset $(75 \mathrm{~s})$ has been added to the $B_{y}$ timeseries to account for the slight longitudinal separation of SOR and the chosen range gate in the high-time resolution beam of the radar. The calculated Hall conductance is insensitive to the uncertainties in this offset $( \pm 20 \%)$ as this uncertainity is small compared to the lifetime of each pulsation ( 20 min). Equivalent currents derived from SOR and BJN are overlaid at appropriate latitudes on the range-time-velocity (RTV) plot (lower panel) Fig. 6. Finally, the calculated Hall conductance is presented (Fig. 6, top panel). All panels are plotted against a common reversed time scale in order to visualise the spatial structure of the omega bands.

Conductance time-series have been calculated for five magnetometer/radar pairs (three magnetometers within the CUTLASS field-of-view, two within the STARE field-of-view) and these are shown in Fig. 7. All CUTLASS derived conductances contain offsets such that the presented profiles occur at positions within beam 9 . STARE derived conductances are calculated using 1-O-S velocities from range cells directly overlaying SOR and TRO.

Beginning with the CUTLASS derived profiles (Fig. 7a), in both the TRO and SOR panels five significant peaks in the Hall conductance are clear. Values of conductance rapidly increase to values $\sim 10$ times above the background level. As the technique employed here to calculate Hall conductance is influenced greatly by the magnitude of the background magnetic field it is very difficult to determine an absolute value of conductance. It is more appropriate to view the presented profiles as indicators of the variability of the ionospheric Hall conductance due to the passage of auroral omega bands through the observation points. Nonetheless sustained conductances of $\sim 100 \mathrm{~S}$ are entirely reasonable, indeed Hall conductances up to $\sim 200 \mathrm{~S}$ have previously been associated with auroral arcs by a number of authors (e.g. Buchert et al., 1988; Lühr and Schlegel, 1994; Aikio and Kaila, 1996). The peaks in conductance at SOR and TRO occur almost simultaneously implying that at scales comparable to the separation of these stations the spatial structure of the omega bands is relatively uniform. By comparing the conductance with derived equivalent currents at both SOR and TRO it can be seen that the Hall conductance increases to its maximum level between the observation of maximum poleward and maximum equatorward equivalent currents. This is consistent with the passage of an upward FAC slightly polewards of the point of observation creating an anticlockwise vortical Hall current (viewed from above) and a region of high conductance due to enhanced electron precipitation. Although the derived conductances at BJN are not as clearly defined as those at lower latitude stations a sequence of successive peaks and troughs are still identifiable. In general, the maximum conductance value at BJN is substantially lower and is observed prior to that at lower latitudes suggesting that the omega band structure is not uniform at all the latitudes observed. More precisely the "leading" of more poleward observations implies that the high conductance/precipitation region is not aligned in a meridional direction. Instead the equatorward region lags behind the poleward by some distance ( $200 \mathrm{~km}$ in the longitudinal direction) as the whole structure propagates eastwards.

Similar conductance signatures of multiple omega band passage are apparent in STARE derived conductances (Fig. 7b). All five omega bands are clearly contained within the STARE/SOR observations. Within the range gate overlaying $\mathrm{TRO}$, no 1-o-s velocity measurements were made after $\sim 03: 15$ UT although four significant conductance intensifications were observed prior to this time. It should be noted that conductances calculated from the STARE beams selected are a factor $\sim 5$ smaller than CUTLASS derived conductances. The selection of beams within the STARE field-of-view that closely overlay SOR and TRO rather than the beam that is most closely aligned to a meridional direction implies that Eq. (4) is no longer accurate as the 1-o-s velocities will be contaminated with the zonal component of the ionospheric flow. Examination of STARE beam 0 (azimuth $\sim 13^{\circ}$ ) reveals that 1 -o-s velocities in a near-meridional direction are $\sim 4$ times smaller than those measured in beams 3 and 4 . Also, the poleward component of velocity, $v_{x}$, measured by STARE is likely to be underestimated due to soundspeed limiting effects (Robinson, 1986). Furthermore, the use of 1-o-s velocities from multiple beams introduces a temporal offset between each beam's estimated conductance profile. It is therefore reasonable to assume that the suppressed conductances and dispersed timings deduced from STARE 1-o-s velocities are artificially distorted due to viewing angle geometry. Once the relevant geometrical issues have been resolved, conduc- 


\section{SUPERDARN PARAMETER PLOT}

11 May $1998^{(131)}$

Finland: vel

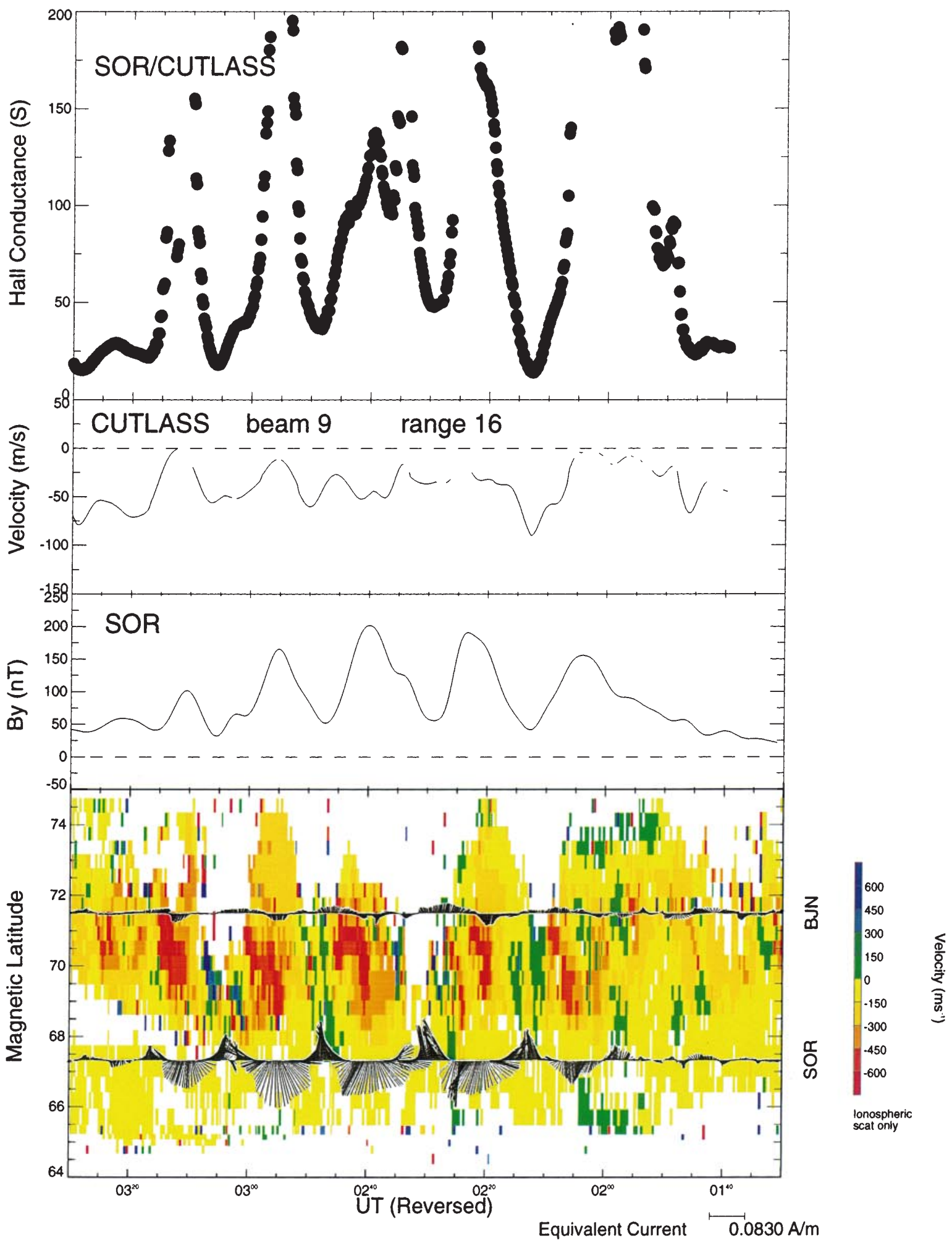


Fig. 6. Line-of-sight velocities from beam 9 of the CUTLASS Finland radar as a function of magnetic latitude and time (bottom panel). Equivalent current patterns deduced from SOR and BJN are overlaid at appropriate latitudes. Filtered magnetometer $Y$ component data from SOR and filtered l-o-s velocity data from CUTLASS beam 9, range 16 are also presented (centre panels). Estimated ionospheric Hall conductances, derived from magnetometer and 1-o-s velocity data is displayed in the top panel (see text for description). All panels are plotted against a common reversed time axis in order to correctly visualise the spatial structure of eastward propagating features

tance magnitudes derived from CUTLASS and STARE are in approximate agreement although direct E-region/ F-region comparisons are not straightforward. Nevertheless the location of regions of elevated Hall conductance at SOR and TRO derived from both CUTLASS and STARE are approximately co-located.

\section{Discussion}

\subsection{Omega band structure}

The observations and interpretation presented so far have been employed to construct a schematic represen-

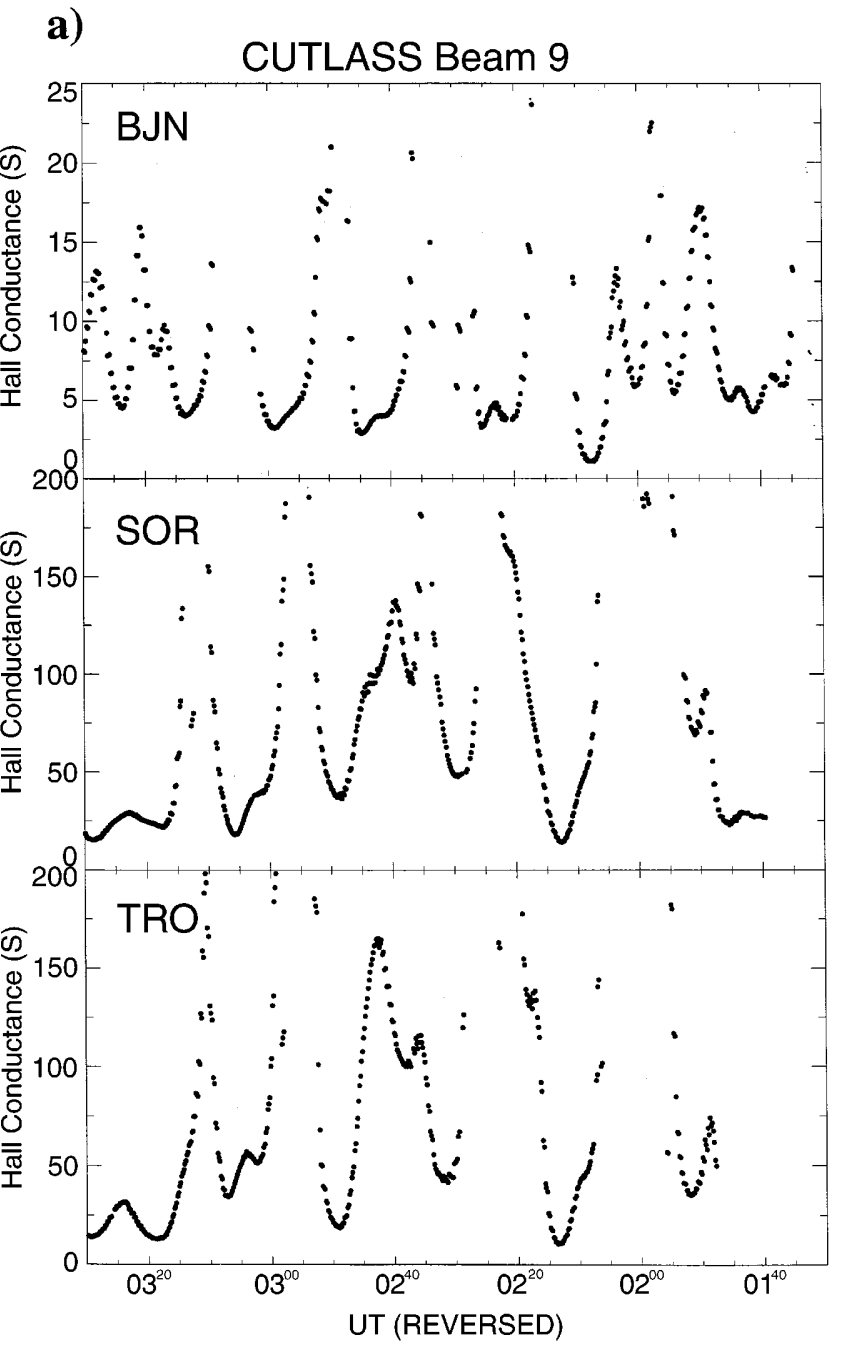

tation of the structure of an auroral omega band (Fig. 8a). Measurements of ionospheric electric field, equivalent currents and derived conductances are combined to infer the optical and current structure of the omega band, associating high conductance with high auroral luminosity. In general the peak of conductance is located on the westward side of the enhanced conductance region that relates to the auroral projection. Highly luminous, high conductance regions are associated with upward FACs (Akasofu, 1981; Opgenoorth et al., 1983), which in this case are located near the poleward boundary of the auroral oval, and around which vortical Hall currents flow in an anticlockwise sense. These upward FACs occur further towards the east at higher latitudes as indicated by the offset of regions of high conductance at $\mathrm{BJN}$ and SOR as shown in Fig. 6. Outside the high conductance region, downward FACs (associated with clockwise Hall currents) allow current closure through the ionosphere. The resulting equatorward current between the two Hall current systems gives rise to the intense poleward flow observed by the radars. This flow is then shifted to higher latitudes by the distorted poleward boundary of the auroral oval as the next omega band moves in from the west. This region is clearly apparent as a diagonal

b)

STARE Beams 3 and 4

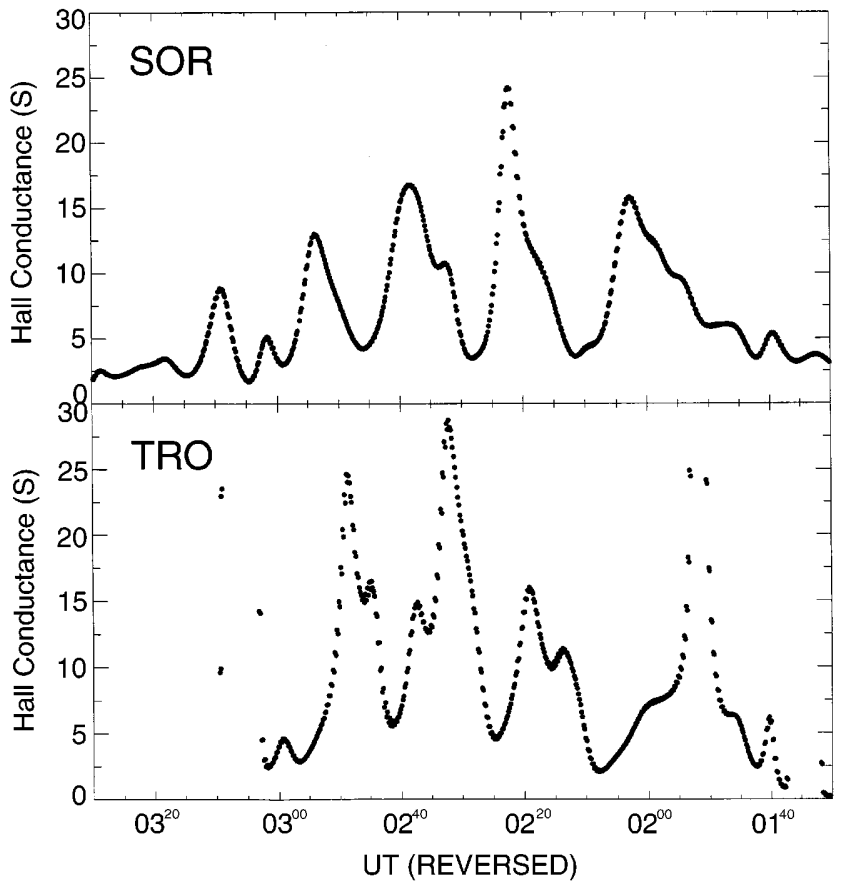

Fig. 7. a Ionospheric Hall conductances derived from CUTLASS beam 9, 1-o-s velocities and IMAGE magnetometer data. Presented are BJN (top panel), SOR (centre panel), and TRO (bottom panel). b Ionospheric Hall conductances derived from STARE beam 4 and SOR (top panel) and STARE beam 3 and TRO (bottom panel). BJN, SOR and TRO are located at magnetic latitudes of $71.3^{\circ}, 67.1^{\circ}$ and $66.4^{\circ}$ respectively. CUTLASS derived profiles are directly comparable as all 1-o-s velocities are drawn from a single beam whereas STARE derived profiles represent simultaneous measurements from two separate locations (see text for description). Conductance scales are not uniform for all panels 


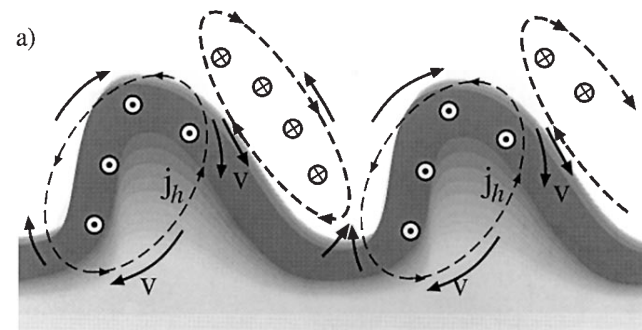

Fully developed auroral $\Omega$ band

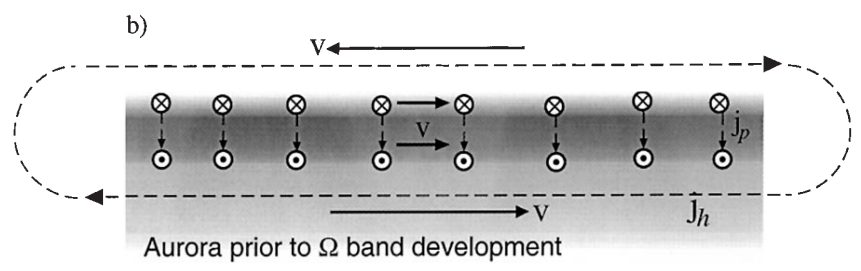

Fig. 8. a Schematic representation of auroral omega bands. Shaded regions represent bright aurora, dashed lines indicate ionospheric Hall currents, heavy solid arrows indicate convection flow direction, the length of the line is an indicator of flow strength. Upward FAC (dotted circles) and downward FAC (crossed circles) are also shown but Pedersen currents have been omitted for clarity. b A simplified interpretation of the region 1-region 2 currents before the advent of instability. Currents, flows and FACs and bright auroral regions are denoted as in a

band of poleward flow in the reversed time scale rangetime-intensity plot in Fig. 6. Finally, just before the high conductance region reaches its maximum poleward extent, vortical Hall currents briefly drive a weak equatorward flow. The scale size of the entire omega band is $\sim 500 \mathrm{~km}$ in the longitudinal direction and $\sim 600 \mathrm{~km}$ in the latitudinal direction. The eastward propagation velocity of the omega bands observed during this interval is $\sim 0.75 \pm 0.1 \mathrm{~km} \mathrm{~s}^{-1}$. This view of omega band structure is consistent with that proposed by Lühr and Schlegel (1994) and it could be considered that a refinement of that description is appropriate to the observations in this case study. Although the EISCAT incoherent scatter radar technique used in that study allows the derivation of $\sum_{H}$ directly from observed ionospheric parameters (i.e. does not require a radar/magnetometer pair and the assumptions required in their combination) the spatial extent of the field-of-view is limited to one location. The multipoint nature of the current study over a much larger field-of-view, albeit with a less absolute method of conductance determination, yields further information regarding the morphology of omega bands.

\subsection{Omega band formation}

The process by which omega bands are created is still unclear. Many authors have linked morning sector auroral omega bands with pairs of field-aligned currents (Opgenoorth et al., 1983; Buchert et al., 1988; Lühr and Schlegel, 1994) although the exact mechanism by which these currents arise remains a subject for debate. The distribution of FACs within the inferred bright region is not consistent with the expected distribution if omega bands were to form as a result of the interchange instability as proposed by Yamamoto et al. (1997). In that case, upward FAC would be concentrated within the bright region at the eastward boundary of each omega band and downward current just inside the westward boundary. Despite the lack of optical observations due to the unsuitable season of the interval presented, the asymmetrical distribution of omega band structure presented here is entirely consistent with optical observations in previous studies (e.g. Snyder and Akasofu, 1974; Opgenoorth et al., 1994). These are characterised by a roughly $\Omega$ shaped distortion of the bright poleward boundary of the auroral oval, slightly darker in the central region than at the edge and often "leaning" slightly in the longitudinal direction. Complementary observations of the ultraviolet aurora routinely made by the Ultra-Violet Imager (UVI) instrument on-board the POLAR spacecraft (Torr et al., 1995) would have proven useful in characterising the spatial structure but these were also unavailable during the interval presented here. The omega bands described here are, however, consistent with the results of a Kelvin-Helmholtz type instability near the poleward boundary of the auroral oval, the mechanism of formation being similar to that of auroral spirals or folds as discussed by Hallinan (1976). In this case a velocity shear would occur at the poleward boundary of the auroral oval in the dawn convection cell where region 1 (downward) and region 2 (upward) FACs lead to Hall currents (opposite in direction to the $\mathbf{E} \times \mathbf{B}$ drifts) as discussed by Fuji et al. (1994) and as indicated in Fig. 8b. It is possible that the presented omega bands have been generated by another instability process but the exact determination of the generation mechanism would require co-ordinated in-situ observations from spacecraft within the appropriate region of the magnetosphere which are unavailable for this interval.

Of particular interest is the relationship of omega bands in the morning sector to substorm phase. Generally observed in the morning hours around 05:00 MLT following substorm activity, it has long been assumed that omega bands are a purely recovery phase phenomenon (Akasofu, 1974; Saito, 1976; Rostoker and Barichello, 1980). The omega bands observed on the morning of 11 May, 1999, occurred during a sequence of substorm onset/intensifications that began at 23:30 UT of the previous day (not shown) with subsequent onset/ intensifications at 00:43, 01:35, 01:50, and 03:05 UT (Fig. 3). The onset/intensification of a substorm to the west over Greenland at 01:50 UT and the almost simultaneous observation (within minutes) of auroral omega bands in the morning sector suggests a more subtle mechanism. The concurrent growth of a magnetospheric substorm during the recovery phase of a previous substorm and the link with auroral omega bands in the morning sector has been discussed previously by Pellinen et al. (1992). Conventional interpretation suggests that the substorm recovery phase is an interval during which the general level of magnetic 
disturbance declines to some quiet level. It seems unlikely then that a system that is tending to a more quiescent state would spontaneously generate a series of instabilities such as omega bands in the morning sector. A new substorm onset, acting upon an already disturbed system could intensify the dawn convection cell, already energised by the previous substorm expansion, and thus could have the ability to drive the large-scale downward currents at the poleward edge of the morning sector oval unstable, creating a series of filamentary FACs. A detailed study of IMAGE network $X$ component magnetograms reveals a sudden intensification of the negative bay at 01:50 UT observed at many stations simultaneously, regardless of longitudinal location. This is in contrast with subsequent observations of Ps 6 pulsations (omega bands) that follows shortly afterwards which are dispersed in longitude. This implies that that the onset of the bay is a global response to the onset/intensification (Opgenoorth and Pellinen, 1998) observed at Greenland and the pulsations are due to drifting omega bands that are formed after the new substorm onset. Furthermore, the absence of Ps 6 pulsations at JAN (located between the Scandinavian and midnight sectors at $70.3^{\circ}$ magnitude latitude) suggests that the observed omega bands have not propagated eastward from the region of substorm onset/intensification but have been created in the morning sector in response to the substorm onset. In order to more fully determine whether or not all omega bands are triggered by an expansion phase onset during the substorm recovery phase, as appears to be the case here, it will be necessary to perform an extensive statistical study of previous omega band intervals and this will be the basis for future research.

\section{Summary}

During the interval 00-08 UT on 11 May, 1998, the CUTLASS Finland HF radar was operating in a high time resolution mode providing $14 \mathrm{~s}$ observations of a single beam in an approximately poleward direction. Between 01:30-03:30 UT a series of five intervals of flow of latitudinal extent $\sim 1.5^{\circ}$ were observed to propagate away from the radar, each lasting for $\sim 10$ min with a repetition period $\sim 18 \mathrm{~min}$. The STARE Norway VHF radar operating in a standard scan mode with temporal resolution $\sim 20 \mathrm{~s}$ also observed extended regions of poleward flow of similar duration and repetition frequency. The latitudinal extent of the enhanced flow region was consistent with that measured by HF radar although STARE observations preceded those from CUTLASS by $\sim 5$ min.

Stations of the IMAGE magnetometer network situated in the Scandinavian sector ( 05:00 MLT) recorded at least five intense Ps 6 pulsation wave-cycles in both the $X$ and $Y$ components between 02:00-03:30 UT implying the nearby transit of a series of auroral omega bands. The scale size of these omega bands has been estimated at $\sim 400 \mathrm{~km}$ in the longitudinal direction and $\sim 600 \mathrm{~km}$ latitude. The delay between observing the pulsations at longitudinally separated stations indicates that the eastward propagation of the omega bands is at a speed of $\sim 0.75 \mathrm{~km} \mathrm{~s}^{-1}$. By studying ionospheric electric fields, ground magnetometer data, derived equivalent currents and derived ionospheric Hall conductances the spatial structure of the observed omega bands has been deduced. The region of elevated conductance has a peak value at the westward edge of each omega bands, and appears to be associated with upward FAC (assumed to be downward precipitating electrons). Downward FACs are located outside of the high conductance regions. The Hall currents that arise as a result of both upward and downward FACs result in strong flows in the region just poleward the auroral boundary between the high conductance regions (inferred to be bright tongues) and weak equatorward flows at the tip of the tongues. This structure is consistent with omega band formation due to a Kelvin-Helmholtz instability near or at the poleward boundary of the auroral oval although observations that would verify this supposition are not available for this interval.

The interval during which the radar observations were made occurred during a period of several magnetospheric substorm onset/intensifications and simultaneously with a new intensification at 01:50 UT observed by the Greenland west coast magnetometer chain located at $\sim 23: 30$ MLT. It is suggested that the global response to a onset/intensification that occurs during the recovery phase of a previous substorm acts as a trigger causing the downward current at the poleward edge of the auroral oval to the east to become unstable. The series of filamentary FACs that would then result manifest themselves as omega bands at the poleward boundary of the auroral oval.

Acknowledgements. We would like to thank Børre Holmeslet, Tromsø Auroral Observatory, for providing the Jan Mayen magnetometer data and the Danish Meteorological Institute for supplying the Greenland magnetometer data. The STARE system is operated jointly by the Max Planck Institute for Aeronomie, Germany, and by the Finnish Meteorological Institute, Finland, in co-operation with SINTEF, University of Trondheim, Norway. The CUTLASS HF radars are deployed and operated by the University of Leicester, and are jointly funded by the UK Particle Physics and Astronomy Research Council, the Finnish Meteorological Institute, and the Swedish Institute of Space Physics. The CUTLASS and STARE radars and the IMAGE magnetometer network all are elements of the Magnetometers - Ionospheric Radars - All sky Cameras Large Experiment (MIRACLE) co-ordinated by the Finnish Meteorological Institute.

The Editor-in-chief thanks A. S. Rodger and another referee for their help in evaluating this paper.

\section{References}

Aikio, A. T., and K. U. Kaila, A substorm observed by EISCAT and other ground-based instruments evidence for near Earth initiation, J. Atmos. Terr. Phys., 58, 5-21, 1996.

Akasofu, S.-I., A study of auroral displays photographed from DMSP-2 satellite and from the Alaska meridian chain of stations, Space Sci. Rev., 16, 617-725, 1974. 
Akasofu, S.-I., Auroral arcs and auroral potential structure, in Physics of Auroral Arc formation, Eds. S.-I. Akasofu and J. R. Kan, American Geophysical Union Geophysical Monograph $25,1981$.

Akasofu, S.-I., and D. S. Kimball, The dynamics of the aurora - I. Instabilities of the aurora, J. Atmos. Terr. Phys., 26, 205-211, 1964.

Buchert, S., W. Baumjohann, G. Haerendel, C. La Hoz, and H. Lühr, Magnetometer and incoherent scatter observations of an intense Ps 6 pulsation event, J. Atmos. Terr. Phys., 50, 357-367, 1988.

Fujii, R., R. A. Hoffman, P. C. Anderson, J. D. Craven, M. Sugiura, L. A. Frank, and N. C. Maynard, Electrodynamic parameters in the nighttime sector during auroral substorms, J. Geophys. Res., 99, 6093-6112, 1994.

Greenwald, R. A., W. Weiss, and E. Nielsen, STARE: a new radar auroral backscatter experiment in northern Scandinavia, Radio Sci., 13, 1021-1039, 1978.

Greenwald, R. A., K. B. Baker, J. R. Dudeney, M. Pinnock, T. B. Jones, E. C. Thomas, J.-P. Villain, J. -C. Cerisier, C. Senior, C. Hanuise, R. D. Hunsucker, G. Sofko, J. Koehler, E. Nielsen, R. Pellinen, A. D. M. Walker, N. Sato, and H. Yamagishi, Darn/ SuperDARN: a global view of the dynamics of high-latitude convection, Space Sci. Rev., 71, 761-796, 1995.

Hallinan, T. J., Auroral spirals: 2. Theory, J. Geophys. Res., 81, 3959-3965, 1976.

Kawasaki, K., and G. Rostoker, Perturbation magnetic fields and current systems associated with eastward drifting auroral structures, J. Geophys. Res., 84, 1464-1480, 1979.

Lühr, H., The IMAGE magnetometer network, STEP Int. Newsl., 4(10), 4-6, 1994.

Lühr, H., and K. Schlegel, Combined measurements of EISCAT and the EISCAT magnetometer cross to study $\Omega$ bands, J. Geophys. Res., 99, 8951-8959, 1994.

Lühr, H., W. Blawert, and H. Todd, The ionospheric plasma flow and current patterns of travelling convection vortices: a case study, J. Atmos. Terr. Phys., 55, 1717-1727, 1993.

Lühr, H., M. Lockwood, P. E. Sandholt, T. L. Hansen, and T. Moretto, Multi-instrument ground-based observations of a travelling convection vortices event, Ann. Geophysicae, 14, 162-181, 1996.

Mravlag, E., M. W. J. Scourfield, A. D. M. Walker, P. R. Sutcliffe, and E. Nielsen, Simultaneous observations of omega band related phenomena in both hemispheres, J. Atmos. Terr. Phys., 53, 309-317, 1991.

Opgenoorth, H. J., and R. J. Pellinen, The reaction of the global convection electrojets to the onset and expansion of the substorm current wedge, Proc. ICS-4, Eds. S. Kokubun and Y. Kamide, Kluwer, Dordrecht, 1998.

Opgenoorth, H. J., J. Oksman, K. U. Kaila, E. Nielsen, and W. Baumjohann, Characteristics of eastward drifting omega bands in the morning sector of the auroral oval, J. Geophys. Res., 88, 9171-9185, 1983.

Opgenoorth, H. J., M. A. L. Persson, T. I. Pulkkinen, and R. J. Pellinen, Recovery phase of magnetospheric substorms and its association with morning sector aurora, J. Geophys. Res., 99, 4115-4129, 1994.

Pellinen, R. J., H. J. Opgenoorth, and T. I. Pulkkinen, Substorm recovery phase: relationship to next activation, Proc. ICS-1, ESA SP-335, 469-475, 1992.

Robinson, T. R., Towards a self-consistent non-linear theory of radar-auroral backscatter, J. Atmos. Terr. Phys., 48, 417-422, 1986.

Rostoker, G., and J. C. Barichello, Seasonal and diurnal variation of Ps 6 magnetic disturbances, J. Geophys. Res., 85, 161-163, 1980.

Rostoker, G., S.-I. Akasofu, J. Foster, R. A. Greenwald, Y. Kamide, K. Kawasaki, A. T. Y. Lui, R. L. McPherron, and C. T. Russell, Magnetospheric substorms - definitions and signatures, J. Geophys. Res., 85, 1663-1668, 1980.

Saito, T., Long-period irregular magnetic pulsations, Pi3, Space Sci. Rev, 21, 427-467, 1978.

Snyder, A. L., Jr., and S. I. Akasofu, Major auroral substorm features in the dark sector observed by a USAF DMSP satellite, Planet. Space Sci., 22, 1511-1517, 1974.

Torr, M. R., D. G. Torr, M. Zukic, R. B. Johnson, J. Ajello, P. Banks, K. Clark, K. Cole, C. Keffer, G. Parks, B. Tsurutani, and J. Spann, A far ultraviolet imager for the international solarterrestrial physics mission, Space Sci. Rev., 71, 329-383, 1995.

Wilhjelm, J., and E. Friis-Christensen, Electric fields and high latitude zonal currents induced by merging of field lines, Geophys. Pap R-31, Dan. Meteorol. Inst., Charlottenlund, Denmark, 1976.

Yamamoto, T., S. Inoue, and C.-I. Meng, Formation of auroral omega bands in the paired region 1 and region 2 field aligned current system, J. Geophys. Res., 102, 2531-2544, 1997. 